\title{
FACTORIZATIONS OF NONNEGATIVE MATRICES
}

\author{
T. L. MARKHAM
}

Abstract. Suppose $A$ is an $n$-square matrix over the real numbers such that all principal minors are nonzero. If $\boldsymbol{A}$ is nonnegative, then necessary and sufficient conditions are determined for $A$ to be factored into a product $L \cdot U$, where $L$ is a lower triangular nonnegative matrix and $U$ is an upper triangular nonnegative matrix with $u_{i i}=1$. These conditions are given in terms of the nonnegativity of certain almost-principal minors of $A$.

I. Introduction. Suppose $A$ is a nonnegative matrix of order $n$ (for which we write $A \geqq 0$ ). The purpose of this paper is to determine necessary and sufficient conditions that $A$ can be factored as $L \cdot U$, where $L$ is a lower triangular nonnegative matrix, and $U$ is an upper triangular nonnegative matrix with $u_{i i}=1$ for $i=1,2, \cdots, n$.

We shall use the following notation. Let $A$ be an $n \times n$ matrix over the field of real numbers. Then $A_{k}$ denotes the principal submatrix of $A$ contained in rows $1,2, \cdots, k$. We indicate the minor of $A$ with rows and columns indexed by $i_{1}, i_{2}, \cdots, i_{p}$ and $j_{1}, j_{2}, \cdots, j_{p}$, respectively, by $A\left(i_{1}, i_{2}, \cdots, i_{p} \mid j_{1}, j_{2}, \cdots, j_{p}\right)$. If $A_{k}$ is nonsingular, the Schur complement of $A_{k}$ in

$$
A=\left(\begin{array}{ll}
A_{k} & A_{12} \\
A_{21} & A_{22}
\end{array}\right)
$$

is $\left(A \mid A_{k}\right)=A_{22}-A_{21} A_{k}^{-1} A_{12}[3]$.

II. $A=L U$ with $L \geqq 0, U \geqq 0$.

THEOREM. Let $A$ be a nonnegative matrix of order $n$ with nonzero principal minors of every order. Then the following statements are equivalent.

(1) $A=L U$, where $L$ is a lower triangular nonnegative matrix, and $U$ is an upper triangular matrix with $u_{i i}=1$.

(2) $\left(A \mid A_{k}\right) \geqq 0$ for $k=1,2, \cdots, n-1$.

(3) $A(1, \cdots, k, i \mid 1, \cdots, k, j) \geqq 0$ for $k=1, \cdots, n-1$ and $k<i, j \leqq n$.

Proof. We demonstrate the following implications: $1 \Rightarrow 2,2 \Rightarrow 3,3 \Rightarrow 1$. Suppose $A=L U$ where

$$
A=\left(\begin{array}{ll}
A_{k} & A_{12} \\
A_{21} & A_{22}
\end{array}\right) .
$$

Received by the editors March 25, 1971 and, in revised form, May 24, 1971.

AMS 1970 subject classifications. Primary 15-00, 15 A48.

Key words and phrases. Factorization, nonnegative matrix, almost principal minor, lower triangular matrix, upper triangular matrix. 
We partition $L$ and $U$ conformably with $A$ as

$$
L=\left(\begin{array}{cc}
L_{11} & 0 \\
L_{21} & L_{22}
\end{array}\right) \text { and } U=\left(\begin{array}{cc}
U_{11} & U_{12} \\
0 & U_{22}
\end{array}\right)
$$

It is immediate that $A_{22}=L_{21} U_{12}+L_{22} U_{22}$; hence $\left(A \mid A_{k}\right)=$ $\left(L U \mid L_{11} U_{11}\right)=L_{21} U_{12}+L_{22} U_{22}-L_{21} U_{11}\left(L_{11} U_{11}\right)^{-1} L_{11} U_{12}=L_{22} U_{22} \geqq 0$ for $k=1,2, \cdots, n-1$.

Next, assume that (2) holds. If $C=\left(A \mid A_{k}\right)=\left(c_{i j}\right)$, then

$$
c_{i j}=\frac{A(1, \cdots, k, i \mid 1, \cdots k, j)}{A(1, \cdots, k \mid 1, \cdots, k)} \geqq 0
$$

for $k=1, \cdots, n-1$ and $k<i, j \leqq n$ by a lemma of Crabtree and Haynsworth [1]. It is easily seen that

$$
A(1, \cdots, k \mid 1, \cdots, k)>0
$$

for $k=1, \cdots, n-1$. (If $k=1$, then $a_{11}>0$. For $k=2$, we have

$$
\frac{A(1,2 \mid 1,2)}{a_{11}} \geqq 0 \text {. }
$$

But $a_{11}>0$, and $A(1,2 \mid 1,2) \neq 0$, so $A(1,2 \mid 1,2)>0$. The procedure is clear for $k=1,2, \cdots, n-1$.) Hence

$$
A(1, \cdots, k, i \mid 1, \cdots, k, j) \geqq 0
$$

for $k=1, \cdots, n-1$ and $k<i, j \leqq n$.

Finally, suppose that (3) is valid. If $n=2$, then

$$
\left(\begin{array}{ll}
a_{11} & a_{12} \\
a_{21} & a_{22}
\end{array}\right)=\left(\begin{array}{cc}
a_{11} & 0 \\
a_{21} & \left(A \mid a_{11}\right.
\end{array}\right)\left(\begin{array}{cc}
1 & \frac{a_{12}}{a_{11}} \\
0 & 1
\end{array}\right) .
$$

so a factorization exists.

Suppose the result holds for $p<n$, and assume $A$ is of order $n$. Now $\left(A \mid a_{11}\right)=\left(m_{i j}\right)$ where

$$
m_{i j}=\frac{A(1, i \mid 1, j)}{a_{11}} \geqq 0
$$

for $1<i, j \leqq n$. Using a well-known identity of Sylvester [2, p. 101], we have

$$
\begin{aligned}
\left(A \mid a_{11}\right)(2, \cdots, k, i \mid 2, \cdots, k, j) & \\
& =\left(1 / a_{11}\right)^{k}\left\{\left(a_{11}\right)^{k-1} A(1, \cdots, k, i \mid 1, \cdots, k, j)\right\} \geqq 0
\end{aligned}
$$


for $2 \leqq k \leqq n-1$ and $k<i, j \leqq n$. Hence, using the inductive hypothesis, there exist $L_{22} \geqq 0$ and $U_{22} \geqq 0$ with $u_{j j}=1$ such that $\left(A \mid a_{11}\right)=L_{22} U_{22}$. Partition

$$
A=\left(\begin{array}{ll}
a_{11} & A_{12} \\
A_{21} & A_{22}
\end{array}\right),
$$

where $A_{22}$ is of order $n-1$. Let $l_{11}=a_{11}, L_{21}=A_{21}$, and $U_{12}=A_{12} / a_{11}$. Then $A_{22}=\left(1 / a_{11}\right) A_{21} A_{12}+L_{22} U_{22}=L_{21} U_{12}+L_{22} U_{22}$. Now if

$$
L=\left(\begin{array}{cc}
l_{11} & 0 \\
L_{21} & L_{22}
\end{array}\right) \text { and } U=\left(\begin{array}{cc}
1 & U_{12} \\
0 & U_{22}
\end{array}\right),
$$

we have $A=L U$ where $L \geqq 0, U \geqq 0$ and $u_{i i}=1$.

The proof of the theorem is now complete.

We would like to observe that there is no loss of generality in (1) in assuming that the diagonal of $U$ consists entirely of 1's. If $A$ has a factorization $L U$ where $L \geqq 0, U \geqq 0$, then let $D=\operatorname{diag}\left(u_{11}, u_{22}, \cdots, u_{n n}\right)$. Then $A=(L D)\left(D^{-1} U\right)=\hat{L} \hat{U}$, where $\hat{L} \geqq 0, \hat{U} \geqq 0$ and $\hat{U}$ has all 1 's on the main diagonal.

The theorem could be stated in an alternative form involving the Schur complement, i.e. $A$ is factorable as $L U$ with $L \geqq 0, U \geqq 0$ if and only if $\left(A \mid a_{11}\right)$ is factorable as $L^{\prime} U^{\prime}$ where $L^{\prime} \geqq 0, U^{\prime} \geqq 0$ with $u_{i i}=1$. Moreover, it is clear that similar results can be obtained for factorizations of the type $U \cdot L$, and these results are independent of each other. For example, if

$$
A=\left(\begin{array}{lll}
2 & 1 & 3 \\
1 & 2 & 2 \\
1 & 2 & 4
\end{array}\right)
$$

then $A=L U$. But $A(1,3 \mid 2,3)<0$, so there is no nonegative factorization of the type $U L$.

\section{REFERENCES}

1. D. E. Crabtree and E. V. Haynsworth, An identity for the Schur complement of a matrix, Proc. Amer. Math. Soc. 22 (1969), 364-366. MR 41 \#234.

2. F. R. Gantmacher, The theory of matrices. Vol. 2, Chelsea, New York, 1959. MR 21 \#6372c.

3. E. V. Haynsworth, Determination of the inertia of a partitioned Hermitian matrix, Linear Algebra and Appl. 1 (1968), no. 1, 73-81. MR 36 \#6440.

Department of Mathematics, University of South Carolina, Columbia, South Carolina 29208 